\title{
Filling in knowledge gaps: Length-weight relations of 46 uncommon sharks and rays (Elasmobranchii) in the Mediterranean Sea
}

\author{
Athanassios C. TSIKLIRAS ${ }^{1}$, Donna DIMARCHOPOULOU ${ }^{1,2}$ \\ 1 Laboratory of Ichthyology, School of Biology, Aristotle University of Thessaloniki, Thessaloniki, Greece \\ 2 Department of Fisheries, Animal and Veterinary Science, University of Rhode Island, Kingston, RI, USA \\ http://zoobank.org/723A6A19-43A5-45B0-AC11-C18EC1710540 \\ Corresponding author: Athanassios C. Tsikliras (atsik@bio.auth.gr)
}

Academic editor: Rodolfo Reyes Jr. • Received 30 December 2020 • Accepted 17 February 2021 • Published 9 September 2021

Citation: Tsikliras AC, Dimarchopoulou D (2021) Filling in knowledge gaps: Length-weight relations of 46 uncommon sharks and rays (Elasmobranchii) in the Mediterranean Sea. Acta Ichthyologica et Piscatoria 51(3): 249-255. https://doi.org/10.3897/ aiep. 51.65858

\begin{abstract}
Large sharks and rays are generally understudied in the Mediterranean Sea, thus leading to a knowledge gap of basic biological characteristics that are important in fisheries management and ecosystem modeling. Out of the 76 sharks and rays inhabiting the Mediterranean Sea, the length-weight relations (LWR) are available for 28 (37\%) of them, usually for common small-sized species that are not protected and may be marketed. The aim of the presently reported study was to fill in the knowledge gap through the estimation of LWR of rare and uncommon sharks and rays in the Mediterranean Sea using the information from single records or few individuals. The analysis was based on a Bayesian hierarchical method for estimating length-weight relations in fishes that has been recently proposed for data-deficient species or museum collections and uses the prior knowledge and existing LWR studies to derive species-specific LWR parameters by body form. The use of this method was applied to single records of rare and uncommon species and here we report the LWR of 46 uncommon sharks and ray species, 14 of which are first reported LWR at a global scale and 21 are the first reported LWR for the Mediterranean Sea; the remaining 11 species are first time records for the western or eastern Mediterranean regions. Museum collections and sporadic catch records of rare emblematic species may provide useful biological information with the use of appropriate Bayesian methods.
\end{abstract}

\section{Keywords}

gap analysis, fisheries management, ecosystems, Mediterranean Sea

\section{Introduction}

According to a recent gap analysis on the biology of Mediterranean fishes, sharks and rays (Class Elasmobranchii) are among the least studied species in the Mediterranean Sea and the lack of knowledge is higher for the less abundant large sharks and rays that are protected and rarely collected in a commercial catch or even scientific surveys (Dimarchopoulou et al. 2017). Despite their conservation status, many sharks are still illegally targeted for their fins and these records are not officially reported (Clarke et al. 2006). Due to the scarcity of samples, the records on the biology of sharks and rays in the Mediterranean are sporadic (Dulvy et al. 2016; Dimarchopoulou et al. 2017), however, because of the importance of large sharks and rays in marine ecosystems and their conservation status (Dulvy et al. 2014), there are several single-catch records available of emblematic species such as the great white shark Carcharodon carcharias (Linnaeus, 1758) (see Kabasakal 2020). The majority of Mediterranean records 
originate from Tunisia and Turkey owing to the lifetime commitment of a few scientists (e.g., Capapé et al. 2006; Kabasakal 2020 among others) and also, in the case of Tunisia, to the higher species richness of sharks and rays in the western Mediterranean Sea (Dulvy et al. 2016). The majority of biological characteristics of small and common sharks - e.g., lesser spotted dogfish, Scyliorhinus canicula (Linnaeus, 1758) - and rays - e.g., thornback ray, Raja clavata Linnaeus, 1758 - that are marketed and not protected in the Mediterranean Sea are better studied (Froese and Pauly 2020).

Length-weight relations (LWR) of fishes (Froese 2006) are important in stock assessments for converting length measurements to weight and estimation of stock biomass (Froese et al. 2011). The LWR is the most studied biological characteristic and studies are available for a large proportion of fish stocks and the majority of finfish in the Mediterranean Sea (Dimarchopoulou et al. 2017). However, rare sharks and rays are underrepresented (Froese and Pauly 2020) and some of the existing LWR records deviate from the cube law dynamics (Froese 2006) because of the small sample size and narrow length and weight distributions (Froese et al. 2011).

A Bayesian hierarchical method for estimating lengthweight relations in fishes has been proposed for data-deficient species that uses the prior knowledge and existing LWR studies to derive species-specific LWR parameters by body form (Froese et al. 2014). According to this method, the approximate values of the slope $(b)$ and the intercept $(a)$ of LWR have been estimated for all fish species and are available in FishBase (Froese and Pauly 2020). Recently, a new approach for estimating LWR from single the length and weight records has been proposed and applied to preserved museum specimens for species for which LWR are not available in the literature (Hay et al. 2020). Based on this approach the LWR of several uncommon fish species, for which museum records are available but their LWR were not, were generated (Hay et al. 2020).

Overall, out of the 43 species of sharks and 33 species of rays that are present in the Mediterranean Sea (Dulvy et al. 2016), LWR are available for 28 species (37\%) based on FishBase records (Froese and Pauly 2020) and the literature (updated dataset of Dimarchopoulou et al. 2017). The aim of the presently reported study was to combine these two approaches and generate LWR for 46 rare shark and ray species in the Mediterranean Sea based on single records (or records of a few individuals) that were available in the literature and for which LWR are lacking at a global or regional scale. Thus, the gap between available and desired knowledge on the LWR of Mediterranean marine fishes will be minimized to only a handful of species.

\section{Methods}

We collected the single specimen records (or few specimens) of total length $(L)[\mathrm{cm}]$ and total weight $(W)$ [g] data for 46 uncommon shark and ray species in the Mediterranean Sea that belong to 10 orders and 16 families from the literature. Then, we estimated their LWR (Table 1) using the Bayesian estimate available for the species based on LWR of taxonomically related species (Froese and Pauly 2020). The number of specimens ranged from a single (17 cases) and double specimens (10 cases) to 27 specimens of the great white shark that are available in a recently published book (Kabasakal 2020). The mean number of specimens was 3.65. All length data were converted to $\mathrm{cm}$ of total length and all weight data to $\mathrm{g}$ of total weight.

In species for which a range of sizes was not available to estimate the parameters of LWR, such as with museum specimens, Hay et al. (2020) assumed that the LWR were isometric, i.e., that the parameter $b=3$, which is a good approximation in the majority of cases (Froese 2006). A similar approach was followed in the presently reported study with $b$ approximated based on related species or species with similar body form (Froese and Pauly 2020) following a Bayesian hierarchical method (Froese et al. 2014).

When total length $(L)$ and total weight $(W)$ measurements from single specimen were available, the parameter $a$ was estimated as (Hay et al. 2020):

$$
a=\frac{W}{L^{b}}
$$

where $b$ is the approximation based on the Bayesian estimate available for the species based on LWR of related species (Froese and Pauly 2020) or body form when a Bayesian estimate was not available (Froese et al. 2014).

When several specimens $(n)$ were available, but not enough to support a valid LWR, because of a small sample size or narrow length range, the mean value of parameter $a$ was estimated as (Hay et al. 2020):

$$
a=\frac{\sum_{i=1}^{n} \times \frac{W}{L^{b}}}{n}
$$

where $b$ is the approximation based on the Bayesian estimate available for the species based on LWR of related species (Froese and Pauly 2020) or body form when a Bayesian estimate was not available (Froese et al. 2014).

\section{Results}

The LWR of 46 uncommon sharks and ray species are reported for the Mediterranean Sea based on published sources of stranded or incidentally caught animals; none of the specimens was preserved or in a museum collection. For 14 species this is the first reported LWR at a global scale and for 21 species this is the first reported LWR for the Mediterranean Sea (Table 1). The remaining 11 species are first-time LWR records for the western (nine species), eastern Mediterranean (one species) regions, and Aegean Sea (one species). 
Table 1. Selected biometric data for 46 species of sharks and rays in the Mediterranean Sea.

\begin{tabular}{|c|c|c|c|c|c|c|c|c|c|c|}
\hline Scientific name & $N$ & $\begin{array}{l}\text { Total length } \\
{[\mathrm{cm}]}\end{array}$ & $\begin{array}{c}\text { Total weight } \\
{[\mathrm{g}]}\end{array}$ & $b$ & $\begin{array}{c}b \\
\text { source }\end{array}$ & $a$ & $a$ range & First LWR & Country & Reference \\
\hline \multicolumn{11}{|l|}{ CARCHARHINIFORMES } \\
\hline \multicolumn{11}{|l|}{ Carcharhinidae } \\
\hline Prionace glauca (Linnaeus, 1758) & 1 & 288.0 & 174000 & 3.11 & BFB & 0.0039 & - & Mediterranean & - & Kohler et al. 2002 \\
\hline Carcharhinus altimus (Springer, 1950) & 2 & $65.2-68.0$ & $2200-3100$ & 3.12 & BFB & 0.0054 & $0.0048-0.0059$ & Mediterranean & Turkey & $\begin{array}{l}\text { Ayas et al. 2020, Turan et } \\
\text { al. } 2020\end{array}$ \\
\hline Carcharhinus brachyurus (Günther, 1870) & 1 & 253.0 & 200000 & 3.09 & BFB & 0.0075 & - & Mediterranean & Italy & Storai et al. 2007 \\
\hline $\begin{array}{l}\text { Carcharhinus brevipinna (Müller et } \\
\text { Henle, 1839) }\end{array}$ & 1 & 115.0 & 11500 & 3.07 & BFB & 0.0054 & - & Mediterranean & Turkey & Ayas et al. 2019 \\
\hline $\begin{array}{l}\text { Carcharhinus falciformis (Müller et } \\
\text { Henle, 1839) }\end{array}$ & 1 & 209.0 & 48000 & 3.09 & BFB & 0.0033 & - & Mediterranean & Italy & Garibaldi and Orsi-Relini 2012 \\
\hline Carcharhinus plumbeus (Nardo, 1827) & 6 & $89.0-300.0$ & $3500-70000$ & 3.17 & BFB & 0.0019 & $0.0010-0.0023$ & Mediterranean & Tunisia & $\begin{array}{l}\text { Capape et al. } 2018 \text {, Soufi et } \\
\text { al. } 2018\end{array}$ \\
\hline $\begin{array}{l}\text { Galeocerdo cuvier (Péron et Lesueur, } \\
\text { 1822) }\end{array}$ & 2 & $95.8-97.4$ & $2750-2840$ & 3.15 & BFB & 0.0016 & $0.0015-0.0016$ & Mediterranean & Libya & Tobuni et al. 2016 \\
\hline \multicolumn{11}{|l|}{ ECHINORHINIFORMES } \\
\hline \multicolumn{11}{|l|}{ Echinorhinidae } \\
\hline Echinorhinus brucus (Bonnaterre, 1788) & 6 & $170.0-254.0$ & $45000-300000$ & 3.12 & BFB & 0.0074 & $0.0031-0.0147$ & Global & $\begin{array}{l}\text { Algeria, } \\
\text { Turkey }\end{array}$ & $\begin{array}{l}\text { Hemida and Capape 2002, } \\
\text { Kabasakal and Bilecenoglu } \\
2014\end{array}$ \\
\hline \multicolumn{11}{|l|}{ HEXANCHIFORMES } \\
\hline \multicolumn{11}{|l|}{ Hexanchidae } \\
\hline Heptranchias perlo (Bonnaterre, 1788) & 5 & $70.0-110.0$ & $1000-5000$ & 3.11 & BFB & 0.0022 & $0.0016-0.0035$ & western Med & $\begin{array}{l}\text { Tunisia, } \\
\text { Spain }\end{array}$ & $\begin{array}{l}\text { El Kamel-Moutalibi et al. } \\
\text { 2014, Guallart et al. } 2019\end{array}$ \\
\hline Hexanchus griseus (Bonnaterre, 1788) & 17 & $250.0-600.0$ & $\begin{array}{l}200000- \\
1000000\end{array}$ & 3.04 & $\mathrm{BF}$ & 0.0062 & $0.0034-0.0123$ & Global & Turkey & Kabasakal 2006 \\
\hline Hexanchus nakamurai Teng, 1962 & 1 & 230.0 & 85000 & 3.11 & BFB & 0.0038 & - & Mediterranean & Albania & Bakiu et al. 2018 \\
\hline \multicolumn{11}{|l|}{ LAMNIFORMES } \\
\hline \multicolumn{11}{|l|}{ Alopiidae } \\
\hline Alopias vulpinus (Bonnaterre, 1788) & 1 & 395.0 & 180000 & 2.86 & BFB & 0.0067 & - & Mediterranean & Turkey & Erguden et al. 2015 \\
\hline Alopias superciliosus Lowe, 1841 & 7 & $151.0-450.0$ & $10000-180000$ & 2.91 & BFB & 0.0051 & $0.0027-0.0068$ & Mediterranean & $\begin{array}{l}\text { Many } \\
\text { countries }\end{array}$ & $\begin{array}{l}\text { Kabasakal and Karhan 2007, } \\
\text { Clo et al. 2008, Damalas and } \\
\text { Megalofonou 2012, Kabasakal } \\
\text { et al. 2011, Lanteri et al. } 2017\end{array}$ \\
\hline \multicolumn{11}{|l|}{ Cetorhinidae } \\
\hline Cetorhinus maximus (Gunnerus, 1765) & 1 & 236.0 & 70000 & 3.04 & $\mathrm{BF}$ & 0.0043 & - & Mediterranean & Turkey & Bilecenoglu et al. 2013 \\
\hline \multicolumn{11}{|l|}{ Lamnidae } \\
\hline Isurus oxyrinchus Rafinesque, 1810 & 1 & 69.8 & 2285 & 3.03 & BFB & 0.0059 & - & Mediterranean & Turkey & Bilecenoglu et al. 2013 \\
\hline Lamna nasus (Bonnaterre, 1788) & 5 & $91.0-236.0$ & $35000-120000$ & 3.03 & BFB & 0.0240 & $0.0077-0.0753$ & Mediterranean & Italy & Storai et al. 2005 \\
\hline Carcharodon carcharias (Linnaeus, 1758) & 27 & $132.0-642.0$ & $\begin{array}{c}27650- \\
2500000\end{array}$ & 3.05 & BFB & 0.0074 & $0.0043-0.0108$ & Mediterranean & Tunisia & $\begin{array}{l}\text { Saidi et al. } 2005 \text {, Morey et } \\
\text { al. } 2003\end{array}$ \\
\hline \multicolumn{11}{|l|}{ Odontaspididae } \\
\hline Carcharias taurus Rafinesque, 1810 & 1 & 99.7 & 3318 & 3.03 & BFB & 0.0029 & - & Mediterranean & Turkey & Ismen et al. 2009 \\
\hline Odontaspis ferox (Risso, 1810) & 2 & $190.0-250.0$ & $34000-180000$ & 3.04 & $\mathrm{BF}$ & 0.0066 & $0.0040-0.0092$ & Mediterranean & Turkey & Kabasakal and Bayri 2019 \\
\hline \multicolumn{11}{|l|}{ MYLIOBATIFORMES } \\
\hline \multicolumn{11}{|l|}{ Dasyatidae } \\
\hline Bathytoshia centroura (Mitchill, 1815) & 1 & 60.1 & 5200 & 3.07 & BFB & 0.0180 & - & western Med & Croatia & Dulcic et al. 2003 \\
\hline Dasyatis chrysonota (Smith, 1828) & 1 & $36.9-44.5$ & $149-445$ & 3.07 & BFB & 0.0030 & $0.0023-0.0039$ & Global & Israel & Golani and Capape 2004 \\
\hline Dasyatis marmorata (Steindachner, 1892) & 1 & 33.0 & 171.8 & 3.07 & BFB & 0.0037 & - & Global & Greece & Chatzispyrou et al. 2020 \\
\hline $\begin{array}{l}\text { Himantura leoparda Manjaji-Matsumoto } \\
\text { et Last, } 2008\end{array}$ & 2 & $26.0-135.2$ & $722-55000$ & 3.17 & BFB & 0.0166 & $0.0097-0.0236$ & Global & Turkey & Yucel et al. 2017 \\
\hline Himantura uarnak (Gmelin, 1789) & 3 & $140.0-148.6$ & $40000-150000$ & 3.17 & BFB & 0.0117 & $0.0047-0.0195$ & Mediterranean & Turkey & $\begin{array}{l}\text { Basusta et al. } 1998 \text {, Ali et } \\
\text { al. } 2010\end{array}$ \\
\hline $\begin{array}{l}\text { Taeniura grabata (Geoffroy Saint- } \\
\text { Hilaire, 1817) }\end{array}$ & 4 & $60.0-117.8$ & $1681-16600$ & 3.17 & BFB & 0.0040 & $0.0033-0.0045$ & Global & Turkey & $\begin{array}{l}\text { Basusta et al. } 1998 \text {, Ali et } \\
\text { al. } 2013\end{array}$ \\
\hline \multicolumn{11}{|l|}{ Mobulidae } \\
\hline Mobula japanica (Müller et Henle, 1841) & 2 & $97.5-120.0$ & $67000-100000$ & 3.04 & BFB & 0.0540 & $0.0478-0.0602$ & Global & Tunisia & Capape et al. 2015 \\
\hline Mobula mobular (Bonnaterre, 1788) & 3 & $200.0-291.5$ & $\begin{array}{c}100000- \\
105000\end{array}$ & 3.04 & BFB & 0.0052 & $0.0022-0.0101$ & Global & $\begin{array}{l}\text { Italy, } \\
\text { Turkey }\end{array}$ & $\begin{array}{l}\text { Scacco et al. 2009, Basusta } \\
\text { and Ozbek } 2017\end{array}$ \\
\hline \multicolumn{11}{|l|}{ Myliobatidae } \\
\hline $\begin{array}{l}\text { Aetomylaeus bovinus (Geoffroy Saint- } \\
\text { Hilaire, 1817) }\end{array}$ & 2 & $152.7-160.0$ & $14800-16200$ & 3.04 & $\mathrm{BF}$ & 0.0033 & $0.0032-0.0034$ & Western Med & Tunisia & El Kamel et al. 2010 \\
\hline Myliobatis aquila (Linnaeus, 1758) & 4 & $50.0-114.0$ & $2000-29400$ & 3.09 & BFB & 0.0111 & $0.0096-0.0130$ & Western Med & France & Capape et al. 2006 \\
\hline RAJIFORMES & & & & & & & & & & \\
\hline Rajidae & & & & & & & & & & \\
\hline Leucoraja melitensis (Clark, 1926) & 1 & 27.0 & 80.2 & 3.13 & BFB & 0.0027 & - & Global & Tunisia & Ben Amor et al. 2018 \\
\hline Dipturus nidarosiensis (Storm, 1881) & 4 & $24.0-148.2$ & $20.12-13783$ & 3.24 & BFB & 0.0010 & $0.0009-0.0013$ & Global & Italy & Follesa et al. 2012 \\
\hline Dipturus oxyrinchus (Linnaeus, 1758) & 1 & 48.0 & 443 & 3.25 & BFB & 0.0015 & - & Western Med & France & Capape et al. 2006 \\
\hline Leucoraja circularis (Couch, 1838) & 6 & $61.9-101.0$ & $1250-5650$ & 3.08 & BFB & 0.0052 & $0.0038-0.0087$ & Western Med & Tunisia & Mnasri et al. 2009 \\
\hline Leucoraja fullonica (Linnaeus, 1758) & 11 & $19.0-76.0$ & $191-2300$ & 3.13 & BFB & 0.0065 & $0.0019-0.0240$ & Global & Italy & Zupa et al. 2010 \\
\hline Leucoraja naevus (Müller et Henle, 1841) & 2 & $22.0-52.0$ & $59-864$ & 3.10 & BFB & 0.0041 & $0.0041-0.0041$ & Mediterranean & Spain & Valls et al. 2011 \\
\hline Raja brachyura Lafont, 1871 & 1 & 91.5 & 5450 & 3.27 & BFB & 0.0021 & - & Eastern Med & France & Capape et al. 2006 \\
\hline Raja undulata Lacepède, 1802 & 2 & $48.4-58.0$ & $765-1356$ & 3.20 & BFB & 0.0031 & $0.0031-0.0031$ & Mediterranean & France & Capape et al. 2006 \\
\hline RHINOPRISTIFORMES & & & & & & & & & & \\
\hline Glaucostegidae & & & & & & & & & & \\
\hline Glaucostegus halavi (Forsskål, 1775) & 1 & 102.3 & 3005 & 2.99 & BFB & 0.0029 & - & Global & Tunisia & Ben Souissi et al. 2007 \\
\hline
\end{tabular}

Table 1 continues on next page. 
Table 1. cont.

\begin{tabular}{|c|c|c|c|c|c|c|c|c|c|c|}
\hline Scientific name & $N$ & $\begin{array}{l}\text { Total length } \\
{[\mathrm{cm}]}\end{array}$ & $\begin{array}{c}\text { Total weight } \\
{[\mathrm{g}]}\end{array}$ & $b$ & $\begin{array}{c}b \\
\text { source }\end{array}$ & $a$ & $a$ range & First LWR & Country & Reference \\
\hline \multicolumn{11}{|l|}{ Rhinopteridae } \\
\hline $\begin{array}{l}\text { Rhinoptera marginata (Geoffroy Saint- } \\
\text { Hilaire, 1817) }\end{array}$ & 5 & $38.5-87.4$ & 104-9980 & 3.10 & BFB & 0.0044 & $0.0010-0.0096$ & Western Med & Turkey & Basusta et al. 2012 \\
\hline \multicolumn{11}{|l|}{ SQUALIFORMES } \\
\hline \multicolumn{11}{|l|}{ Oxynotidae } \\
\hline Oxynotus centrina (Linnaeus, 1758) & 4 & $53.3-79.0$ & $1649-5020$ & 3.04 & $\mathrm{BF}$ & 0.0087 & $0.0080-0.0093$ & Aegean Sea & Greece & $\begin{array}{l}\text { Kousteni and Megalofonou } \\
2016\end{array}$ \\
\hline \multicolumn{11}{|l|}{ SQUATINIFORMES } \\
\hline \multicolumn{11}{|l|}{ Squatinidae } \\
\hline Squatina aculeata Cuvier, 1829 & 1 & 79.9 & 3690 & 3.04 & BFB & 0.0061 & - & Global & Turkey & Basusta 2002 \\
\hline Squatina oculata Bonaparte, 1840 & 6 & $29.1-79.5$ & $173-3750$ & 3.04 & BFB & 0.0067 & $0.0061-0.0076$ & Global & $\begin{array}{l}\text { Greece, } \\
\text { Italy }\end{array}$ & $\begin{array}{l}\text { Corsini and Zava 2007, Zava } \\
\text { et al. 2016, Erguden et al. } 2019\end{array}$ \\
\hline Squatina squatina (Linnaeus, 1758) & 2 & $38.0-156.0$ & $1900-32600$ & 3.02 & BFB & 0.0200 & $0.0078-0.0322$ & Mediterranean & Turkey & $\begin{array}{c}\text { Akyol et al. 2015, Cavallaro } \\
\text { et al. } 2015\end{array}$ \\
\hline \multicolumn{11}{|l|}{ TORPEDINIFORMES } \\
\hline \multicolumn{11}{|l|}{ Torpedinidae } \\
\hline Tetronarce nobiliana (Bonaparte, 1835) & 4 & $17.0-102.0$ & $150-20000$ & 2.96 & BFB & 0.0256 & $0.0133-0.0342$ & Western Med & France & Capape et al. 2006 \\
\hline Torpedo marmorata Risso, 1810 & 4 & $16.0-50.0$ & $40-3500$ & 2.94 & $\mathrm{BFB}$ & 0.0241 & $0.0115-0.0354$ & Western Med & France & Capape et al. 2006 \\
\hline Torpedo torpedo (Linnaeus, 1758) & 2 & $25.0-39.0$ & $300-1012$ & 2.90 & BFB & 0.0255 & $0.0246-0.0265$ & Mediterranean & France & Capape et al. 2006 \\
\hline
\end{tabular}

A Bayesian estimate of parameter $b$ based on LWR of related species was available for 41 species (Froese and Pauly 2020), while for the remaining 5 ones-Hexanchus griseus (Bonnaterre, 1788), Cetorhinus maximus (Gunnerus, 1765), Odontaspis ferox (Risso, 1810), Aetomylaeus bovinus (Geoffroy Saint-Hilaire, 1817), Oxynotus centrina (Linnaeus, 1758) — an estimate based on their body form was used (Froese et al. 2014). In cases where more than one specimen was available, the range of length, weight, and parameter $a$ is provided (Table 1).

\section{Discussion}

Although isometric growth $(b=3)$ is the most common type of growth in the majority of families (Froese 2006), strong deviations from isometry have been observed due to a narrow range of sizes and/or low sample size (Froese et al. 2011; Hay et al. 2020). In addition, as the seasonality in $b$ values is strong along with the corresponding estimates of $a$ (Froese 2006), a limited sampling period, even with a large sample size of the entire somatic range of the species may lead to varying LWR (Hay et al. 2020). This effect of seasonality is partly related to the spawning period of fishes and it is especially strong for the female viviparous sharks that deviate a lot in somatic weight during their gestation period (Castro 2000).

Length data for a few specimens of some other very rare shark species exist in the literature-pigeye shark, Carcharhinus amboinensis (Müller et Henle, 1839) (see Da Maddalena and Della Rovere 2005); common sawfish, Pristis pristis (Linnaeus, 1758) (see Capape et al. 2006); dusky shark, Carcharhinus obscurus (Lesueur, 1818) (see Bilecenoglu et al. 2013)_-but weight data are lacking as those specimens were spotted at fish markets, where only part of the body was "available" usually the head, or are based on photos or anecdotal records from newspapers and magazines. Anecdotal evidence and historical records may be really valuable in reconstructing the history of these iconic predators (Ferretti et al. 2016) and gaining insight into the previous status of marine ecosystems and ecosystem effects of fishing (Pauly 1995; Pauly et al. 1998).

This work extends the approach of gaining valuable information from museum collections (Hay et al. 2020) to rare and sporadic catch records of emblematic species such as large sharks and rays for which biological information is lacking, at least in the Mediterranean Sea. The importance of the Bayesian approach to data-deficient areas and species is highlighted along with the need for recording the basic biological information (length, weight, and sex) even from single specimens of rare and uncommon sharks and rays. As the majority of these species are rare and protected (although illegally landed in many areas of the Mediterranean Sea), sometimes such data are only available in fish markets and newspapers/ magazines; nowadays also through social media (Kabasakal and Bilecenoglu 2020).

\section{Acknowledgments}

The authors would like to thank Daniel Pauly and Rainer Froese for their insightful suggestions and two anonymous reviewers for their helpful comments.

\section{References}

Akyol O, Ünal V, Capapé C (2015) Occurrence and biological observations on angel shark Squatina squatina (Chondrichthyes: Squatinidae) from the Turkish waters (eastern Mediterranean). Turkish Journal of Fisheries and Aquatic Sciences 15: 925-929. https://doi. org/10.4194/1303-2712-v15_4_17
Ali M, Saad A, Ben Amor MM, Capapé C (2010) First records of the honeycomb stingray, Himantura uarnak (Forskål, 1775), off the Syrian coast (eastern Mediterranean) (Chondrichthyes: Dasyatidae). Zoology in the Middle East 49(1): 104-106. https://doi.org/10.1080 /09397140.2010.10638397 
Ali M, Saad A, Reynaud C, Capapé C (2013) First records of the round fantail stingray, Taeniura grabata (Chondrichthyes: Dasyatidae), off the Syrian coast (eastern Mediterranean). Zoology in the Middle East 59(2): 176-178. https://doi.org/10.1080/09397140.2013.810883

Ayas D, Çiftçi N, Akbora HD (2019) New Record of Carcharhinus brevipinna (Müller \& Henle, 1839) from Mersin Bay, the Northeastern Mediterranean. NESciences 4: 268-275. https://doi.org/10.28978/ nesciences. 646334

Ayas D, Çiftçi N, Yalcin E, Akbora HD, Bakan M, Erguden D (2020) First record of the big nose shark, Carcharhinus altimus (Springer, 1950) from Mersin Bay. International Journal of Fisheries and Aquatic Studies 8: 132-136

Bakiu R, Cakalli M, Giovos I (2018) The first record of bigeyed sixgill shark, Hexanchus nakamurai Teng, 1962 in Albanian waters. Journal of the Black Sea/Mediterranean Environment 24: 74-79.

Basusta N (2002) Occurrence of a sawback angelshark (Squatina aculeata Cuvier, 1829) off the Eastern Mediterranean Coast of Turkey. Turkish Journal of Veterinary and Animal Sciences 26: 1177-1179.

Basusta N, Ozbek EO (2017) New record of giant devil ray, Mobula mobular (Bonnaterre, 1788) from the Gulf of Antalya (Eastern Mediterranean Sea). Journal of the Black Sea/Mediterranean Environment 22: 162-169.

Basusta N, Erdem U, Kumlu M (1998) Two new fish records for the Turkish seas: Round stingray Taeniura grabata and skate stingray Himantura uarnak (Dasyatidae). Israel Journal of Zoology 44: 65-66.

Basusta A, Ozer EI, Sulikowski JA, Basusta N (2012) First record of a gravid female and neonate of the Lusitanian cownose ray, Rhinoptera marginata, from the eastern Mediterranean Sea. Journal of Applied Ichthyology 28(4): 643-644. https://doi.org/10.1111/j.14390426.2012.01941.x

Ben Amor MM, Ounifi Ben-Amor K, Capapé C (2018) Record of a critically endangered skate, Leucoraja melitensis (Chondrichthyes: Rajidae) from Tunisian coast (central Mediterranean). Thalassia Salentina 40: 9-16. https://doi.org/10.1285/i15910725v40p9

Ben Souissi J, Golani D, Mejri H, Ben Salem M, Capapé C (2007) First confirmed record of the Halave's guitarfish, Rhinobatos halavi (Forsskål, 1775) (Chondrichthyes: Rhinobatidae) in the Mediterranean Sea with a description of a case of albinism in elasmobranchs. Cahiers de Biologie Marine 48: 67-75.

Bilecenoglu M, Alfaya J, Azzurro E, Baldacconi R, Boyaci Y, Circosta V, Compagno L, Coppola F, Deidun A, Durgham H, Durucan F, Ergüden D, Fernández-Álvarez F, Gianguzza P, Giglio G, Gökoğlu M, Gürlek M, Ikhtiyar S, Kabasakal H, Karachle P, Katsanevakis S, Koutsogiannopoulos D, Lanfranco E, Micarelli P, Özvarol Y, Pena-Rivas L, Poursanidis D, Saliba J, Sperone E, Tibullo D, Tiralongo F, Tripepi S, Turan C, Vella P, Yokeş M, Zava B (2013) New Mediterranean marine biodiversity records (December, 2013). Mediterranean Marine Science 14(2): 463-480. https://doi. org/10.12681/mms.676

Capapé C, Guélorget O, Vergne Y, Marqués A, Quignard J-P (2006) Skates and rays (Chondrichthyes) from waters off the Languedocian coast (southern France, northern Mediterranean). Annales, Series Historia Naturalis 16: 166-178.

Capapé C, Rafrafi-Nouira S, El Kamel-Moutalibi O, Boumaïza M, Reynaud C (2015) First Mediterranean records of spinetail devil ray, Mobula japanica (Elasmobranchii: Rajiformes: Mobulidae). Acta Ichthyologica et Piscatoria 45(2): 211-215. https://doi.org/10.3750/ AIP2015.45.2.13
Capapé C, Rafrafi-Nouira S, Ounifi Ben-Amor K, Ben Amor MM (2018) Additional records of sandbar shark, Carcharhinus plumbeus (Chondrichthyes: Carcharhinidae) from the northern Tunisian coast (central Mediterranean Sea). Annales, Series Historia Naturalis 28: 99-104. https://doi.org/10.19233/ASHN.2018.11

Castro JI (2000) The biology of the nurse shark, Ginglymostoma cirratum, off the Florida east coast and the Bahama Islands. Environmental Biology of Fishes 58(1): 1-22. https://doi. org/10.1023/A:1007698017645

Cavallaro M, Danze A, Ammendolia G, Navarra E (2015) Finding of a rare Squatina squatina (Linnaeus, 1758) (Chondrichthyes: Squatinidae) along the Tyrrhenian coast of the Strait of Messina and its maintenance in an aquarium. Marine Biodiversity Records 8: e44. https://doi.org/10.1017/S1755267215000226

Chatzispyrou A, Gubili C, Laiaki M, Mantopoulou-Palouka D, Kavadas S (2020) First record of the marbled ray, Dasyatis marmorata (Elasmobranchii: Dasyatidae), from Greece (central Aegean Sea). Biodiversity Data Journal 8: e51100. https://oi.org/10.3897/ BDJ.8.e51100

Clarke SC, McAllister MK, Milner-Gulland EJ, Kirkwood GP, Michielsens CGJ, Agnew DJ, Pikitch EK, Nakano H, Shivji MS (2006) Global estimates of shark catches using trade records from commercial markets. Ecology Letters 9(10): 1115-1126. https://doi. org/10.1111/j.1461-0248.2006.00968.x

Clo S, Bonfil R, de Sabata E (2008) Additional records of the bigeye thresher shark, Alopias superciliosus, from the central and eastern Mediterranean Sea. Marine Biodiversity Records 2: e20. https://doi. org/10.1017/S1755267208000237

Corsini M, Zava B (2007) Recent capture of Squatina oculata and Squatina aculeata from Dodecanese Islands (SE Aegean Sea, eastern Mediterranean). Biologia Marina Mediterranea 14: 352-353.

Da Maddalena A, Della Rovere G (2005) First record of pigeye shark, Carcharhinus amboinensis in the Mediterranean Sea. Annales, Series Historia Naturalis 15: 209-212.

Damalas D, Megalofonou P (2012) Occurrences of large sharks in the open waters of the southeastern Mediterranean Sea. Journal of Natural History 46(43-44): 2701-2723. https://doi.org/10.1080/002 22933.2012.716864

Dimarchopoulou D, Stergiou KI, Tsikliras AC (2017) Gap analysis on the biology of Mediterranean marine fishes. PLoS ONE 12(4): e0175949. https://doi.org/10.1371/journal.pone.0175949

Dulcic J, Jardas I, Onofri V, Bolotin J (2003) The roughtail stingray Dasyatis centroura (Pisces: Dasyatidae) and spiny butterfly ray Gymnura altavela (Pisces: Gymnuridae) from the southern Adriatic. Journal of the Marine Biological Association of the United Kingdom 83(4): 871-872. https://doi.org/10.1017/S0025315403007926h

Dulvy NK, Fowler SL, Musick JA, Cavanagh RD, Kyne PM, Harrison LR, Carlson JK, Davidson LN, Fordham SV, Francis MP, Pollock CM, Simpfendorfer CA, Burgess GH, Carpenter KE, Compagno LJ, Ebert DA, Gibson C, Heupel MR, Livingstone SR, Sanciangco JC, Stevens JD, Valenti S, White WT (2014) Extinction risk and conservation of the world's sharks and rays. eLife 3: e00590. https://doi. org/10.7554/eLife.00590

Dulvy NK, Allen DJ, Ralph GM, Walls RHL (2016) The conservation status of sharks, rays and chimaeras in the Mediterranean Sea. IUCN, Malaga, Spain.

El Kamel O, Mnasri N, Boumaiza M, Ben Amor MM, Raynaud C, Capapé C (2010) Additional records of the bull ray, Pteromylaeus bovinus (Chondrichthyes: Myliobatidae) in the lagoon of Bizerte 
(northern Tunisia, central Mediterranean). Annales, Series Historia Naturalis 20: 169-174.

El Kamel-Moutalibi O, Mnasri-Sioudi N, Rafrafi-Nouira S, Boumaiza M, Raynaud C, Capapé C (2014) Additional records of a rare elasmobranch species, sharpnose seven-gill shark Heptranchias perlo (Hexanchidae) off the northern Tunisian coast (central Mediterranean). Annales, Series Historia Naturalis 24: 99-106.

Erguden D, Gurlek M, Turan C (2015) Occurrence of the Thresher Alopias vulpinus (Bonnaterre, 1788) from the Northeastern Mediterranean coast of Turkey. Biharean Biologist 9: 76-77.

Erguden D, Ayas D, Gurlek M, Karan S, Turan C (2019) First documented smoothback angelshark Squatina oculata Bonaparte, 1840 from the north-eastern Mediterranean Sea, Turkey. Cahiers de Biologie Marine 60: 189-194. https://doi.org/10.21411/CBM.A.23607FF9

Ferretti F, Morey Verd G, Seret B, Sulić Šprem J, Micheli F (2016) Falling through the cracks: The fading history of a large iconic predator. Fish and Fisheries 17(3): 875-889. https://doi.org/10.1111/ faf. 12108

Follesa MC, Cannas R, Cabiddu S, Cau A, Mulas A, Porcu C, Cau A (2012) Preliminary observations of the reproductive biology and diet for the Norwegian skate Dipturus nidarosiensis (Rajidae) from the central western Mediterranean Sea. Cybium 36: 473-477. https://oi.org/10.26028/cybium/2012-363-006

Froese R (2006) Cube law, condition factor, and weight-length relationships: History, meta-analysis and recommendations. Journal of Applied Ichthyology 22(4): 241-253. https://doi.org/10.1111/j.14390426.2006.00805.x

Froese R, Pauly D (2020) FishBase. https://www.fishbase.org

Froese R, Tsikliras AC, Stergiou KI (2011) Editorial note on weightlength relations of fishes. Acta Ichthyologica et Piscatoria 41(4): 261-263. https://doi.org/10.3750/AIP2011.41.4.01

Froese R, Thorson JT, Reyes Jr RB (2014) A Bayesian approach for estimating length-weight relationships in fishes. Journal of Applied Ichthyology 30(1): 78-85. https://doi.org/10.1111/jai.12299

Garibaldi F, Orsi-Relini L (2012) Record of Carcharhinus falciformis (Bibron in Müller \& Henle, 1839), in Italian waters (Ligurian Sea, Northwestern Mediterranean). Cybium 36: 399-400. https://doi. org/10.26028/cybium/2012-362-011

Golani D, Capapé C (2004) First records of the blue stingray, Dasyatis chrysonota (Smith, 1828) (Chondrichthyes: Dasyatidae), off the coast of Israel (eastern Mediterranean). Acta Adriatica 45: 107-113.

Guallart J, Morey G, Bartoli A (2019) New record of a sharpnose sevengill shark Heptranchias perlo (Elasmobranchii, Hexanchidae) from the Balearic Sea, western Mediterranean Sea. Journal of Fish Biology 94(3): 526-531. https://doi.org/10.1111/jfb.13905

Hay A, Xian W, Bailly N, Liang C, Pauly D (2020) The why and how of determining length-weight relationships of fish from preserved museum specimens. Journal of Applied Ichthyology 36(3): 373-379. https://doi.org/10.1111/jai.14014

Hemida F, Capapé C (2002) Observations on a female bramble shark, Echinorhinus brucus (Bonnaterre, 1788) (Chondrichthyes: Echinorhinidae), caught off the Algerian coast (southern Mediterranean). Acta Adriatica 43: 103-108.

Ismen A, Yigin CC, Altinagac U, Ayaz A (2009) Length-weight relationships for ten shark species from Saros Bay (North Aegean Sea). Journal of Applied Ichthyology 25(S1): 109-112. https://doi. org/10.1111/j.1439-0426.2009.01263.x
Kabasakal H (2006) Distribution and biology of the bluntnose sixgill shark, Hexanchus griseus (Bonnaterre, 1788) (Chondrichthyes: Hexanchidae), from Turkish waters. Annales, Series Historia Naturalis $16: 29-36$.

Kabasakal H (2020) Agreement with the monster-Lessons we learned from the great white shark in Turkish waters. Turkish Marine Research Foundation (TUDAV) Publication No: 57, Istanbul, Turkey, 74 pp.

Kabasakal H, Bayri E (2019) Notes on the occurrence of smalltooth sandtiger shark, Odontaspis ferox (Lamniformes: Odontaspididae) from Antalya Bay, eastern Mediterranean, Turkey. Journal of the Black Sea/Mediterranean Environment 25: 166-171.

Kabasakal H, Bilecenoglu M (2014) Not disappeared, just rare! status of the bramble shark, Echinorhinus brucus (Elasmobranchii: Echinorhinidae) in the seas of Turkey. Annales, Series Historia Naturalis 24: 93-98.

Kabasakal H, Bilecenoglu M (2020) Shark infested internet: An analysis of internet-based media reports on rare and large sharks of Turkey. FishTaxa 16: 8-18.

Kabasakal H, Karhan SU (2007) On the occurrence of the bigeye thresher shark, Alopias superciliosus (Chondrichthyes: Alopiidae), in Turkish waters. Marine Biodiversity Records 1: e69. https://doi. org/10.1017/S1755267207007452

Kabasakal H, Dalyan C, Yurtsever A (2011) Additional records of the bigeye thresher shark Alopias superciliosus (Lowe, 1839) (Chondrichthyes: Lamniformes: Alopiidae) from Turkish waters. Annales, Series Historia Naturalis 21: 143-148.

Kohler NE, Turner PA, Hoey JJ, Natanson LJ, Briggs R (2002) Tag and recapture data for three pelagic shark species: blue shark (Prionace glauca), shortfin mako (Isurus oxyrinchus), and porbeagle (Lamna nasus) in the North Atlantic Ocean. Collected Volumes of the Scientific Papers of ICCAT 54: 1231-1260.

Kousteni V, Megalofonou P (2016) Observations on the biological traits of the rare shark Oxynotus centrina (Chondrichthyes: Oxynotidae) in the Hellenic seas. Journal of Fish Biology 89(3): 1880-1888. https://doi.org/10.1111/jfb.13077

Lanteri L, Castellano L, Garibaldi F (2017) New record of Alopias superciliosus Lowe, 1841 in the north-western Mediterranean and annotated review of the Mediterranean records. Acta Adriatica 58(2): 313-324. https://doi.org/10.32582/aa.58.2.10

Mnasri N, Boumaïza M, Capapé C (2009) Morphological data, biological observations and occurrence of a rare skate, Leucoraja circularis (Chondrichthyes: Rajidae), off the northern coast of Tunisia (central Mediterranean). Pan-American Journal of Aquatic Sciences 4: 70-78.

Morey G, Martinez M, Massuti E, Moranta J (2003) The occurrence of white sharks, Carcharodon carcharias, around the Balearic Islands (western Mediterranean Sea). Environmental Biology of Fishes 68(4): 425-432. https://doi.org/10.1023/B:EBFI.0000005789.83761.d8

Pauly D (1995) Anecdotes and the shifting baseline syndrome of fisheries. Trends in Ecology and Evolution 10(10): 430-430. https://doi. org/10.1016/S0169-5347(00)89171-5

Pauly D, Christensen V, Dalsgaard J, Froese R, Torres Jr F (1998) Fishing down marine food webs. Science 279(5352): 860-863. https:// doi.org/10.1126/science.279.5352.860

Saidi B, Bradai MN, Bouain A, Guelorget O, Capapé C (2005) Capture of a pregnant female white shark, Carcharodon carcharias 
(Lamnidae) in the Gulf of Gabès (southern Tunisia, central Mediterranean) with comments on oophagy in sharks. Cybium 29: 303-307.

Scacco U, Consalvo I, Dimuccio S, Tunesi L (2009) On the by-catch of two porbeagle sharks Lamna nasus in the central Adriatic Sea. Marine Biodiversity Records 5: e61. https://doi.org/10.1017/S1755267212000127

Soufi-Kechaou E, Ounifi Ben-Amor K, Ben Souissi J, Ben Amor MM, Capapé C (2018) The capture of a large predatory shark, Carcharhinus plumbeus (Chondrichthyes: Carcharhinidae), off the Tunisian coast (central Mediterranean). Annales, Series Historia Naturalis 28: 23-28.

Storai T, Celona A, Zuffa M, De Maddalena A (2005) On the occurrence of the porbeagle, Lamna nasus (Bonnaterre, 1788) (Chondrichthyes: Lamnidae), off Italian coasts (northern and central Mediterranean Sea): A historical survey. Annales, Series Historia Naturalis 15: 195-202.

Storai T, Zinzula L, Cristo B, Human BA (2007) First record of Carcharhinus brachyurus (Gunther, 1870) (Chondrichthyes; Carcharhinidae) from Sardinian waters (central Mediterranean). Annales, Series Historia Naturalis 17: 177-182.

Tobuni IM, Benabdallah RB, Serena F, Shakman EA (2016) First documented presence of Galeocerdo cuvier (Péron \& Lesueur, 1822) (Elasmobranchii, Carcharhinidae) in the Mediterranean basin
(Libyan waters). Marine Biodiversity Records 9(1): e94. https://doi. org/10.1186/s41200-016-0089-3

Turan C, İğde M, Ergenler A, Ayas D (2020) New record of the bignose shark, Carcharhinus altimus (Springer, 1950) (Carcharhiniformes: Carcharhinidae), in Turkish waters. Journal of the Black Sea/Mediterranean Environment 26: 119-125.

Valls M, Quetglas A, Ordines F, Moranta J (2011) Feeding ecology of demersal elasmobranchs from the shelf and slope off the Balearic Sea western Mediterranean. Scientia Marina 75(4): 633-639. https://doi.org/10.3989/scimar.2011.75n4633

Yucel N, Sakalli A, Karahan A (2017) First record of the honeycomb stingray Himantura leoparda (Manjaji-Matsumoto \& Last, 2008) (Myliobatoidei: Dasyatidae) in the Mediterranean Sea, confirmed by DNA barcoding. Journal of Applied Ichthyology 33(3): 530-532. https://doi.org/10.1111/jai.13283

Zava B, Fiorentino F, Serena F (2016) Occurrence of juveniles Squatina oculata Bonaparte, 1840 (Elasmobranchii: Squatinidae) in the Strait of Sicily (central Mediterranean). Cybium 40(4): 341-343. https:// doi.org/10.26028/cybium/2016-404-011

Zupa W, Donnaloia M, Gaudio P, Intini S, Carbonara P (2010) Occurrence of Leucoraja fullonica (Linnaeus, 1758) in the south Adriatic Sea. Biologia Marina Mediterranea 17: 260-261. 